\title{
Comparative Serum Proteomic Analysis of Differentially Regulated Proteins in Patients with Rheumatoid Arthritis and Healthy Volunteers
}

\author{
Radovan Vasko ${ }^{1 *}$, Sabine Blaschke ${ }^{2}$, Jan-Hendrik Streich ${ }^{1}$, Gerhard A Müller ${ }^{1}$, Peter Korsten ${ }^{1}$ and Hassan Dihazi ${ }^{1}$ \\ ${ }^{1}$ Department of Nephrology and Rheumatology, University Medical Center Goettingen, 37075 Goettingen, Germany \\ ${ }^{2}$ Interdisciplinary Emergency Care Unit, University Medical Center Goettingen, 37075 Goettingen, Germany
}

*Corresponding author: Radovan Vasko, Department of Nephrology and Rheumatology, University Medical Center Goettingen, Robert-Koch-Str. 40 , 37075 Göttingen, Germany, Tel: 49-551-39-9825; E-mail: vaskorad@gmail.com

Rec date: May 12, 2016; Acc date: June 07, 2016; Pub date: June 11, 2016

Copyright: (c) 2016 Vasko R, et al. This is an open-access article distributed under the terms of the Creative Commons Attribution License, which permits unrestricted use, distribution, and reproduction in any medium, provided the original author and source are credited.

\begin{abstract}
Background: To identify differentially regulated serum proteins, we compared proteome profiles of sera from patients with rheumatoid arthritis (RA) and healthy controls using proteomics.

Methods: Sera were collected from 43 patients with RA and 48 healthy volunteers. The samples were cleared of the most abundant major proteins by immunoaffinity chromatography. Serum protein profiles between the two groups were compared by two-dimensional differential gel electrophoresis (2D-DIGE) and differentially regulated proteins were studied using mass spectrometry.

Results: We identified 26 differentially expressed serum proteins between patients with RA and healthy controls. A quantitatively significant change of protein levels was defined as at least 1.5-fold upregulation or 0.6-fold downregulation respectively. Using these criteria, patients with RA exhibited significantly higher levels of leucine-rich alpha-2-glycoprotein $(p<0.01)$, apolipoprotein A-IV $(p<0.001)$, clusterin $(p<0.001)$, haptoglobin $(p<0.001)$, Ig alpha-1 chain $C$ region $(p<0.05)$, retinol-binding protein $4(p<0.001)$, serum amyloid $A(p<0.01)$ and alpha-1-antichymotrypsin $(p<0.01)$. The levels of serotransferrin were significantly decreased in RA patients $(p<0.01)$.

Conclusion: We identified eight proteins with significantly increased and one protein with significantly decreased serum levels in RA patients compared to healthy controls. Several of these proteins may be implicated in the pathogenesis of RA and may have potential in diagnostics and activity assessment of RA.
\end{abstract}

Keywords: Rheumatoid arthritis; Serum proteins; Proteomics

\section{Introduction}

Rheumatoid arthritis (RA) is the most common type of chronic autoimmune inflammatory joint disease. The traditional diagnostic approach based on elevated inflammatory markers, such as erythrocyte sedimentation rate and C-reactive protein (CRP) has been complemented by more specific tests for rheumatoid factor and anticyclic citrullinated peptide antibodies. A timely diagnosis is of particular importance in patients presenting with early RA. We still encounter patients with arthritis who are challenging to diagnose due to the atypical presentation and/or the lack of characteristic laboratory or radiological findings. Because of the limited sensitivity and specificity of the established biomarkers, it is of great significance to identify additional candidate proteins, which may improve diagnostics and also increase understanding of the mechanisms underlying RA. Proteomic technologies together with bioinformatics tools have successfully been employed for identification of such diagnostic indicators [1]. The objective of the present study was a group comparison of the protein profiles between RA patients and healthy controls using two-dimensional differential gel electrophoresis and peptide mass spectrometric analyses.

\section{Material and Methods}

\section{Serum depletion and protein precipitation}

All RA patients included in the study met the criteria for RA established by the American College of Rheumatology. Patients who suffered from other chronic diseases or acute infections within the preceding 3 months were excluded. None of the volunteers had previous history of arthralgia or other chronic disease. Equal amounts of sera obtained from each patient with RA or healthy control were pooled and depleted for high abundant proteins using two different HPLC-based systems, Agilent Human 6 (Agilent, Boeblingen, Germany) and BC ProteomeLab IgY-12 LC2 (Beckman Coulter, Krefeld, Germany). The depletion resulted in the elimination of albumin, immunoglobulin G (IgG), immunoglobulin A (IgA), antitrypsin, transferrin, haptoglobin and the subsequent precipitation of proteins was carried out as described previously [1].

\section{Two-dimensional gel electrophoresis (2-DE)}

The 2-DE was performed as described previously [2]. The 2-DE gels were stained with Flamingo fluorescent gel stain (Bio-Rad, Hercules, CA, USA) according to the manufacturer's instructions. After staining, gels were digitized using a Fuji FLA-5100 scanner (Fuji Photo, Kanagawa, Japan) and the images were analyzed with Delta2D software (Decodon, Brunswick, Germany). For protein visualization, 
Citation: Vasko R, Blaschke S, Streich JH, Müller GA, Korsten P, et al. (2016) Comparative Serum Proteomic Analysis of Differentially Regulated Proteins in Patients with Rheumatoid Arthritis and Healthy Volunteers. J Arthritis 5: 201. doi:10.4172/2167-7921.1000201

Page 2 of 4

the gels were additionally stained overnight with colloidal Coomassie blue (Roti-Blue, Roth, Karlsruhe, Germany).

\section{Two-dimensional differential gel electrophoresis (2D-DIGE)}

2D-DIGE was carried out as described previously [2]. The CyDye labeled gels were scanned using a Fuji FLA-5100 scanner, spot matching across gels and normalization based on the internal standard was performed with Delta2D software. To analyze the significance of protein regulation, a Student's t test was performed, and statistical significance was assumed for p-values less than 0.05 . For protein visualization, the 2 -DE gels were stained overnight with colloidal Coomassie blue. Differentially regulated proteins were excised and processed for identification by mass spectrometry.

\section{Protein identification by mass spectrometry}

Manually excised gel plugs were subjected to in gel digestion for the identification of gel-separated proteins. The mass spectrometric analysis was performed as earlier described resulting in confident protein identifications [3]. Database searches in the Swiss-Prot and NCBI primary sequence database were performed using the MASCOT Software (Matrix Science, London, UK).

\section{Results}

Serum samples were collected from 43 patients with RA (32 women and 11 men, mean age 55.3 years) and 48 healthy volunteers (22 women and 26 men, mean age 34.9 years). The sera were depleted for high abundant proteins, which obstruct signals from less abundant, however, more important proteins in terms of searching for putative disease biomarkers. To assure, that the expected differences in protein expression are not simply a consequence of the depletion procedure, we implemented two different depletion systems (Agilent Human 6 and BC ProteomeLab) and analyzed patients' sera in parallel.

Firstly, in order to obtain a general overview of the serum proteome, the depleted sera were separated with a standard 2-DE and proteins were identified using mass spectrometry. A total of 103 spots were visualized in the gel, which resulted in identification of 62 nonredundant proteins (Supplementary Figure 1 and Supplementary Table $1)$.

Serum proteins have abundant post-translational modifications and therefore, quantitative comparisons of samples based on standard 2DE may produce inconsistent results. The advantage of 2D-DIGE is that both samples (each labelled with a different fluorescent dye), RA and control serum run in a single gel under the same experimental conditions, which eliminates inconsistencies between gels and greatly improve quantification and statistical validity compared to standard 2DE.

The reference map of the serum proteome indicated that $81 \%$ of the 103 spots lie within the $\mathrm{pH}$ range 4-7. Hence, we scrutinized this specific region in more detail using zooming 2D-DIGE (Figure 1). For each depletion system (Agilent Human 6 and BC ProteomeLab) and patient group (RA and control), four replicate gels were obtained. As a result, the comparative 2D-DIGE proteome analysis revealed with high statistical confidence 26 differentially expressed serum proteins between RA patients and healthy controls (Figure 2). A quantitatively significant increase or decrease of protein expression was defined as at least 1.5-fold upregulation or 0.6-fold downregulation respectively, and the expression change has been simultaneously confirmed by both approaches (Agilent Human 6 and BC ProteomeLab). The fold difference of significantly upregulated or downregulated proteins is presented below as the average the expression obtained using the two approaches (Agilent Human 6 and BC ProteomeLab).

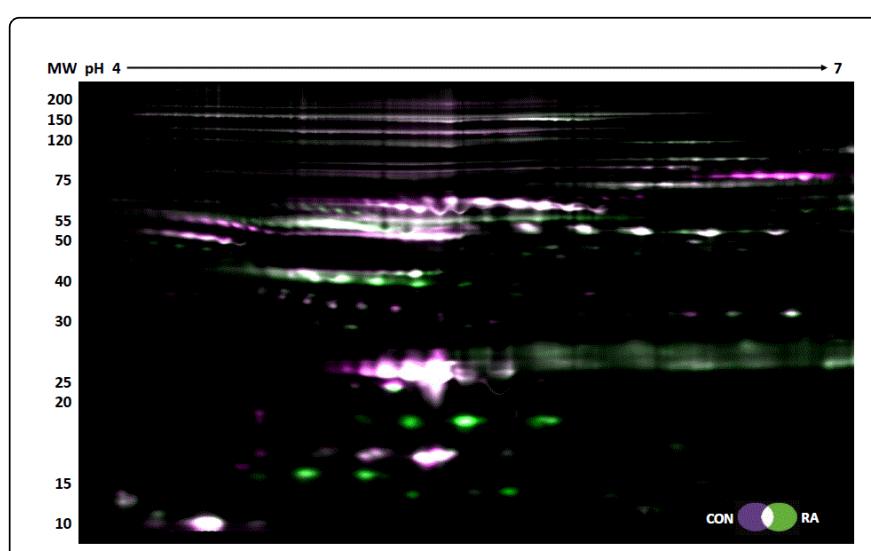

Figure 1: Representative two-dimensional differential gel electrophoresis (2D-DIGE) image of serum proteins from patients with rheumatoid arthritis (RA) and healthy controls (CON). The pooled serum samples were immunodepleted using Agilent Human 6 multiple affinity removal column. Fifty micrograms of depleted serum protein from RA patients and healthy controls were labelled with CyDye DIGE Fluor minimal dyes according to the manufacturer's protocol (GE Healthcare, USA). The labelled gel was scanned using a Fuji FLA-5100 scanner. 12\% Criterion ${ }^{\bullet}$ Bis-Tris Neutralgel pH 4-7 (Bio-Rad)

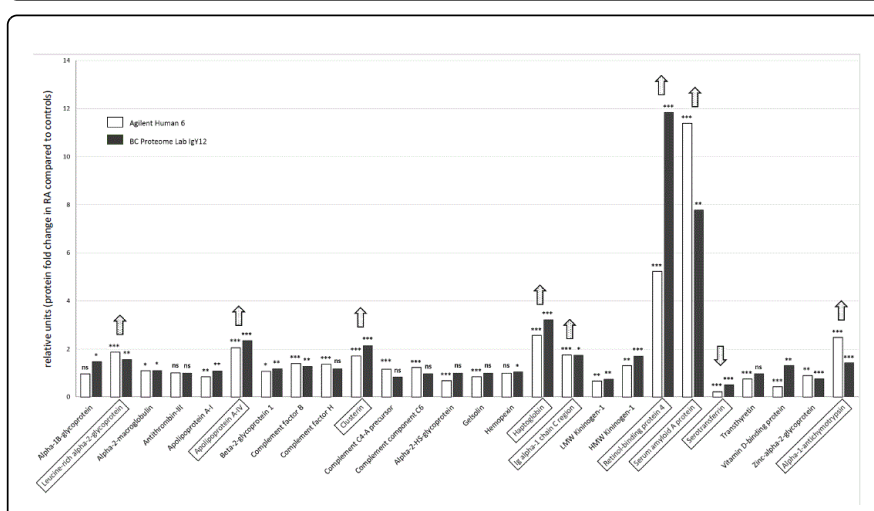

Figure 2: Comparative proteome analysis identified 26 differentially expressed serum proteins between patients with rheumatoid arthritis (RA) or healthy controls. The graphs demonstrate differentially expressed serum proteins in patients with RA compared to healthy controls. The results are presented on the $y$ axis as the ratio of the spot volume reflecting the fold change in RA patients compared to the control group (RA/control). For each protein, the expression difference obtained using both, Agilent Human 6 (white columns) and BC ProteomeLab (dark columns) system is presented. Proteins which met the criteria of quantitatively significantly increased (at least 1.5 -fold upregulation) or decreased (at least 0.6-fold downregulation) expression are marked with arrows. Statistically significant differences: $\left(^{\star}\right) \mathrm{p}<0.05$, $\left.{ }^{* *}\right) \mathrm{p}<0.01,\left({ }^{* * *}\right) \mathrm{p}<0.001$ 
Patients with RA showed a 1.72-fold increased expression of leucine-rich alpha-2-glycoprotein (LRG, $\mathrm{p}<0.01$ ), a 2.20 -fold increase of apolipoprotein A-IV (APOA4, $\mathrm{p}<0.001$ ), a 1.93-fold increase of clusterin $(p<0.001)$, a 2.89 -fold increase of haptoglobin $(p<0.001)$, a 1.75 -fold increase of Ig alpha-1 chain $\mathrm{C}$ region (IGHA1, $\mathrm{p}<0.05$ ), a 8.53 -fold increase of retinol-binding protein 4 (RBP4, $\mathrm{p}<0.001)$, a 9.59fold increased expression of serum amyloid A (SAA, $p<0.01$ ), and a 1.96-fold upregulation of alpha-1-antichymotrypsin (AAT, $\mathrm{p}<0.001$ ). The expression of serotransferrin was significantly decreased in RA patients (0.37-fold, $\mathrm{p}<0.001 \mathrm{~s})$.

\section{Discussion}

Quantitative proteomic profiling revealed nine differentially regulated proteins in sera from patients with RA. Several of these proteins, like haptoglobin, SAA, AAT or serotransferrin are traditional acute phase proteins and reflect a chronically increased inflammatory status in RA. Here, we briefly discuss the current evidence and potential diagnostic utility of the less conventional proteins that were overexpressed in sera from patients with RA.

LRG is a glycoprotein whose biological function and regulation has not been fully understood. A recent study reported that LRG promotes aberrant angiogenesis through modulation of TGF- $\beta 1$ signaling [4]. Bearing in mind the role of neovascularization in the inflamed synovium, LRG upregulation may represent a relevant molecular mechanism in the pathogenesis of RA. In fact, LRG has been reported as a promising biomarker for monitoring RA disease activity [5]. Whereas patients with apparently inactive RA did not differ in terms of CRP from healthy subjects, serum LRG levels were significantly higher in subjects with RA [6]. This may be particularly valuable in assessment of patients with early RA or in patients with active disease and normal CRP levels.

CLU is a highly conserved chaperone protein with anti-apoptotic and anti-inflammatory properties mediated through inhibition of complement and NF- $\mathrm{KB}$ pathways [7]. There is no study comparing serum CLU levels between RA and healthy subjects. Several studies reported decreased synovial expression of CLU in RA compared to osteoarthritis [8]. Interestingly, patients with systemic sclerosis who have higher serum CLU levels develop less frequently digital ulcers and pulmonary hypertension [9]. Similarly, serum CLU concentration correlated inversely with the disease activity in systemic lupus erythematosus [10].

RBP4 is an adipokine associated with obesity and development of insulin resistance, and subjects with elevated serum RBP4 are at risk for cardiovascular disease [11]. RBP4 stimulates the expression of proinflammatory cytokines in endothelial cells via activation of NADPH oxidase and NF- $\mathrm{KB}$ pathway [12]. The synovial expression of adiponectin, a member of adipokine family was increased RA and stimulation of synovial fibroblasts with adiponectin induced synthesis of proinflammatory cytokines and chemokines [13].

We demonstrated that identification of a single serum protein possessing the characteristics of an "exclusive" RA marker might be indeed very difficult. Our work revealed that several relevant proteins are dysregulated in RA. Despite the progress in this area, further studies are needed to establish and functionally connect these novel proteins in order to elucidate the molecular mechanisms involved in the pathogenesis of rheumatoid arthritis. We did not consider adjustment for use of medications. Our intent was to conduct a study under routine clinic conditions, where we try to establish a diagnosis without asking the patients to interrupt their therapy for the purpose of blood analysis. We believe that a combination of results obtained under "real life" settings with data acquired under meticulously controlled conditions provide the best valuable information for characterization and creation of disease-related protein fingerprints, which hold enormous potential to improve the diagnostics and therapy of RA patients in the future.

\section{Ethics and Consent}

The study was approved by the local Ethics Committee at the University Medical Center in Goettingen and all subjects provided written informed consent prior to participation in the study.

\section{Acknowledgments}

The authors would like to thank Dr. B. Mai (Vitos Orthopedic Clinic, Kassel, Germany), Dr. M. Kostrzewa and Dr. K. Sparbier (both Bruker Daltonics, Bremen, Germany) for valuable comments and suggestions during the study.

\section{References}

1. Blaschke S, Rinke K, Maring M, Flad T, Patschan S, et al. (2015) Haptoglobin-alpha1, -alpha2, vitamin D-binding protein and apolipoprotein C-III as predictors of etanercept drug response in rheumatoid arthritis. Arthritis Res Ther 17: 45.

2. Dihazi H, Dihazi GH, Nolte J, Meyer S, Jahn O, et al. (2009) Multipotent adult germline stem cells and embryonic stem cells: comparative proteomic approach. J Proteome Res 8: 5497-5510.

3. Dihazi GH, Mueller GA, Asif AR, Eltoweissy M, Wessels JT, et al. (2015) Proteomic characterization of adrenal gland embryonic development reveals early initiation of steroid metabolism and reduction of the retinoic acid pathway. Proteome Sci 13: 6

4. Wang X, Abraham S, McKenzie JA, Jeffs N, Swire M, et al. (2013) LRG1 promotes angiogenesis by modulating endothelial TGF-beta signalling. Nature 499: 306-311.

5. Serada S, Fujimoto M, Ogata A, Terabe F, Hirano T, et al. (2010) iTRAQbased proteomic identification of leucine-rich alpha-2 glycoprotein as a novel inflammatory biomarker in autoimmune diseases. Ann Rheum Dis 69: 770-774.

6. Ha YJ, Kang EJ, Lee SW, Lee SK, Park YB, et al. (2014) Usefulness of serum leucine-rich alpha-2 glycoprotein as a disease activity biomarker in patients with rheumatoid arthritis. J Korean Med Sci 29: 1199-1204.

7. Falgarone G, Chiocchia G (2009) Chapter 8: Clusterin: A multifacet protein at the crossroad of inflammation and autoimmunity. Adv Cancer Res 104: 139-170.

8. Devauchelle V, Essabbani A, De Pinieux G, Germain S, Tourneur L, et al. (2006) Characterization and functional consequences of underexpression of clusterin in rheumatoid arthritis. J Immunol 177: 6471-6479.

9. Yanaba K, Asano Y, Tada Y, Sugaya M, Kadono T, et al. (2012) A possible contribution of elevated serum clusterin levels to the inhibition of digital ulcers and pulmonary arterial hypertension in systemic sclerosis. Arch Dermatol Res 304: 459-463.

10. Newkirk MM, Apostolakos P, Neville C, Fortin PR (1999) Systemic lupus erythematosus, a disease associated with low levels of clusterin/apoJ, an antiinflammatory protein. J Rheumatol 26: 597-603.

11. Kotnik P, Fischer-Posovszky P, Wabitsch M (2011) RBP4: a controversial adipokine. Eur J Endocrinol 165: 703-711.

12. Farjo KM, Farjo RA, Halsey S, Moiseyev G, Ma JX (2012) Retinol-binding protein 4 induces inflammation in human endothelial cells by an NADPH oxidase- and nuclear factor kappa B-dependent and retinol-independent mechanism. Mol Cell Biol 32: 5103-5115. 
Citation: Vasko R, Blaschke S, Streich JH, Müller GA, Korsten P, et al. (2016) Comparative Serum Proteomic Analysis of Differentially Regulated Proteins in Patients with Rheumatoid Arthritis and Healthy Volunteers. J Arthritis 5: 201. doi:10.4172/2167-7921.1000201

Page 4 of 4

13. Frommer KW, Zimmermann B, Meier FM, Schroder D, Heil M, et al. (2010) Adiponectin-mediated changes in effector cells involved in the pathophysiology of rheumatoid arthritis. Arthritis Rheum 62: 2886-2899. 